\title{
How to be an atroges DLRO locum ... and be invited back!
}

\section{Dental hygienist Rachael England from Bracknell shares her top tips for securing regular employment as a dental hygienist in your region.}

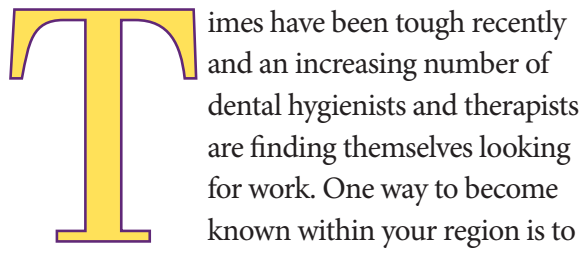

offer your services as a locum.

\section{Agency or independent? \\ Agencies}

There are many dental agencies and building a good relationship with them is essential. Register with as many as possible; you will find one or two with whom you build rapport and work for regularly. Keep them updated: days you're available, feedback from your experiences, changes to your CV. Agencies will also have a good relationship with certain practices, who will regularly ask them to provide a locum.

\section{Independent}

It may take some time to become established as an independent locum. Start by contacting dental practices to let them know you're available and on which days. Send them your $\mathrm{CV}$ and have references prepared. Practices may already have a regular locum or enough clinicians to cover shortfalls, so don't be disheartened if you receive no response.

\section{TOP TIPS}

\section{Stand out from the crowd!}

What marks you out against all the other clinicians looking for work? Have an outstanding CV. Invest time in developing your $\mathrm{CV}$, sell yourself and build on your experiences and competencies - they don't have to be directly professional. Have you been travelling? Have you done volunteer work? What have your experiences taught you as a person? Tailor your $\mathrm{CV}$ to the practice by writing it as though you are the ideal candidate for the job.

As for your covering letter, address it to the principal by name. What do you have to offer them? We all know dentists love being flattered: tell them what appeals to you about their practice ... have you heard of their reputation for outstanding patient care?

Do you have a business card? Quick and easy online business cards enclosed with your CV give practices a handy reference to contact you.

\section{5) Be organised}

So, you have work lined up, either independently or through an agency. It's useful to have a selection of your favourite freshly sharpened instruments and oral hygiene instruction aids in your kit bag. Not to mention clean pressed uniform, loupes, invoice book and lunch money!

Snoop on the Internet: look up the practice you will be visiting. Find out who the dentists and support staff are, what kind of services are provided, the clientele to expect, location and route planner.

Call ahead, speak to the practice manager; introduce yourself and confirm your hours, where you can park, is the practice easy to find, will you be assisted in surgery ... and ask any questions you might have about the practice.

You will need to take your GDC registration, indemnity certificate, Hepatitis B inoculation proof, and CRB certificate.

\section{(3) Be punctual}

(2) Leave extra time to allow for morning traffic and getting lost. Last year I sat outside a practice for 30 minutes waiting for them to open; unfortunately it was the wrong practice! Being late for your first patient gives a terrible impression: you'll have to work extra hard to make up for that.

\section{Be professional and ethical It goes without saying that you treat} patients as you would in your own established practice. Check the prescription, medical history and past notes; get a feel for the type of patient you may be about to meet. What has their oral hygiene regime been in the past? Are they routine hygiene? In periodontal therapy? Maintenance? Do they usually have local anaesthetic? Are there any social notes you can use to quickly gain rapport?

Ensure your notes are thorough and contemporaneous; remember someone else may be treating them at their next visit: they need to know what has happened and the advice given.

I'm sure many practices can tell horror stories of locums turfing the patient out after ten minutes so they can straighten their hair or other outrageous behaviour - don't be one of them.

\section{Introduce yourself \\ (2) Patients don't like change and you may be replacing a very much loved clinician, or they may be seeing another new face after a string of stand-ins. Be polite and introduce yourself and be prepared to answer myriad questions about yourself. Remember you're in charge and steer the conversation onto professional ground.}

\section{Re helpful}

(1) Should you have 'white space' or a 'failed to attend', once you have caught up with your clinical duties make yourself useful. Ask the receptionist or practice manager if they have any little jobs, filing or retrieving cards for example; if not put the kettle on and be sure to offer everyone a cup.

\section{Show interest \\ Have you seen an interesting case? Met} a lovely patient? If you have the opportunity, discuss them with the dentist or practice manager. Motivated and educated clinicians are a real asset to any practice. Are you taking a course? Tell them, ring your own bell; let them know about your passion for your career.

\section{(0) Have respect \\ 0 Leave the surgery stocked up and tidy,} follow and sign the surgery protocols. The permanent clinician will appreciate coming back to a neat surgery and hopefully give good feedback for you.

Remember you're representing an agency and putting your own reputation on the line.

(1) Invoices either BACS payment or a stamped addressed envelope for the principal to easily send me a cheque. Remember as a self-employed clinician the practice has 30 days in which to settle your invoice.

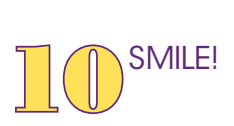

